\title{
Percepção Visual de Contraste em Humanos: Evidência Psicofísica para Canais de Freqüência Angular Alta
}

\author{
Visual Contrast Perception in Human Beings: Psychophysical Evidence \\ for High Angular Frequency Channels
}

\author{
Natanael Antonio dos Santos*a ${ }^{* a}$, Maria Lúcia de Bustamante Simas ${ }^{b}$ \\ \& Renata Maria Toscano Barreto Lyra Nogueira ${ }^{\mathrm{c}}$ \\ ${ }^{a}$ Universidade Federal da Paraíba, João Pessoa, Brasil, ${ }^{b}$ Universidade Federal de Pernambuco, Recife, Brasil, \\ ${ }^{c}$ Universidade de São Paulo, São Paulo, Brasil
}

\begin{abstract}
Resumo
O objetivo deste trabalho foi mensurar curvas de resposta ao contraste para os filtros de freqüências angulares de banda estreita de $32,48,64$ e 96 ciclos $/ 360^{\circ}$. Foram estimadas nove curvas para cada filtro com o método psicofísico de somação de resposta de supralimiar aliado ao método da escolha forçada. Participaram deste experimento seis participantes adultos com acuidade visual normal ou corrigida. Os resultados demonstraram somações máximas de limiar de contraste na freqüência de teste dos filtros de 32, 48, 64 e 96 ciclos $/ 360^{\circ}$ circundadas por inibições nas freqüências vizinhas às freqüências angulares de teste de cada filtro. Estes resultados são consistentes com a existência de filtros de freqüências angulares de banda estreita no sistema visual humano através do processo de somação ou inibição na faixa de freqüência angular alta.

Palavras-chave: Percepção visual da forma; filtro angular; freqüência angular; método psicofísico de somação de supralimiar.

Abstract

The aim of this study was to measure narrow-band frequency response curves for four angular frequency filters. The test frequencies were 32, 48, 64 and 96 cycles $/ 360^{\circ}$. Six humans observers with normal or corrected visual acuity measured nine curves for each filter, with a supra-threshold response summation psychophysical method allied with a forced-choice method. The results showed maximum summation effects at test frequency for filter frequencies $32,48,64$ and 96 cycles $/ 360^{\circ}$, as well as a strong inhibition for neighboring frequencies. These results are consistent with the existence of narrow-band angular frequency filters in the human visual system either through summation or the inhibition of specific high angular frequency ranges.

Keywords: Visual perception of form; angular frequency filter; angular frequency; supra-threshold summation method.
\end{abstract}

Os processos fundamentais da percepção visual da forma ou de contraste são bem estabelecidos e partem da premissa de que os objetos são processados por canais ou mecanismos que respondem seletivamente a faixas diferentes de freqüências espaciais e orientações (Blakemore \& Campbell, 1969a, 1969b; Campbell \& Robson, 1968; R. L. De Valois \& K. K. De Valois, 1988; Sachs, Nachmias \& Robson, 1971). Em outras palavras, as teorias de canais múltiplos se fundamentam no princípio de que durante a percepção visual da forma, uma cena visual qualquer é decomposta em seus componentes mais elementares (freqüências espaciais), os quais o córtex visual utiliza para fazer suas representações (R. L. De Valois \& K. K. De Valois, 1988; O’Donnell et al., 2002). Um canal do ponto de vista psicofísico é, por exemplo, um filtro com estrutura

\footnotetext{
* Endereço para correspondência: Laboratório de Processamento Visual Humano, Departamento de Psicologia, Universidade Federal da Paraíba, João Pessoa, PB, 58051-900. Fone (Fax.): (83) 216-7006. E-mail: natanael_labv@yahoo.com.br; natanael@cchla.ufpb.br

Suporte Financeiro: CNPq, FACEPE.
}

independente no sistema que lida com uma faixa estreita de freqüência espacial. Já do ponto de vista fisiológico, um canal significa uma população neuronal sintonizada para uma banda-estreita de freqüência espacial (Santos \& Simas, 2001a, 2002b).

Vários métodos psicofísicos podem ser utilizados para demonstrar a existência de canais múltiplos de freqüências espaciais, como por exemplo, adaptação, mascaramento, somação de resposta de sublimiar e somação de resposta de supralimiar (R. L. De Valois \& K. K. De Valois, 1988; Santos \& Simas, 2002a). No entanto, as primeiras evidências psicofísicas para canais múltiplos de freqüências espaciais vieram a partir do surgimento da técnica da adaptação seletiva a freqüências espaciais. Essa técnica surgiu de um trabalho de Gilinski (1968) que observou que depois de visualizar um padrão listrado de contraste alto por um período de tempo, observadores podem mostrar um prejuízo para perceber padrões de freqüência espacial e orientação idênticas, mas de contraste mais baixo. Gilinski descreveu esse fenômeno que ficou conhecido como adaptação e que demonstrou ser altamente seletivo para orientação e freqüência 
espacial (Blakemore \& Campbell, 1969a; R. L. De Valois \& K. K. De Valois, 1988; Menees, 1998). Blakemore e Campbell realizaram um dos primeiros e mais convincentes estudos realizados com o método da adaptação seletiva favoráveis à existência de canais múltiplos de freqüências espaciais. Esses autores verificaram que a adaptação à grade senoidal eleva o limiar da freqüência espacial próxima à freqüência adaptada.

Atualmente, estudos psicofísicos em humanos e estudos neurofisiológicos em macacos e gatos fornecem fortes evidências para os modelos teóricos de canais múltiplos ou mecanismos sintonizados para freqüências espaciais (R. L. De Valois \& K. K. De Valois, 1988; Maffei \& Fiorentini, 1973; Menees, 1998; Santos, Simas \& Nogueira, 2003, 2004; Simas \& Santos, 2002a, 2002b; Wilson, Levi, Maffei, Rovamo \& De Valois, 1990). Entretanto, a maioria dos estudos, que procuraram investigar as propriedades dos mecanismos seletivos a freqüências espaciais no sistema visual humano (SVH), utilizou estímulos elementares do tipo grade senoidal vertical em coordenadas cartesianas. A grade senoidal é um dos padrões elementares clássicos mais conhecido e estudado. Esta é derivada da modulação senoidal da luminância ao longo de uma dimensão. Sendo assim, a mesma pode ser facilmente descrita em termos de freqüência espacial que é normalmente expressado pelo o número de ciclos (ou listras claras e escuras) por grau de ângulo visual (cpg). Logo, a grade senoidal é normalmente conhecida ou referendada como representando uma freqüência espacial.

Por outro lado, nas últimas décadas, o interesse dos pesquisadores em caracterizar a resposta do SVH para estímulos diferentes de grade senoidal aumentou. É cada vez maior na literatura a utilização de estímulos de freqüências radiais e angulares em coordenadas polares para descrever o processo operacional realizado pelo sistema visual (Amidor, 1997; Santos \& Simas, 2002b; Simas, Frutuoso \& Vieira, 1992; Simas \& Santos, 2002a, 2002b; Verrall \& Kakarala, 1998; Wilson \& Wilkinson, 1997, 1998). De certa forma, estes estudos foram impulsionados com os achados eletrofisiológicos de Gallant e colaboradores, que demonstraram que células simples na área visual V4 de macaco têm preferências para padrões radias e angulares em coordenadas polares (Gallant, Brau \& van Essen, 1993; Gallant, Connor, Rakshit, Lewis \& van Essen, 1996).

Matematicamente, as modulações senoidais ou cossenoidais que definem um estímulo elementar podem ser descritas tanto por um sistema de coordenadas cartesianas como por um sistema de coordenadas polares. A diferença entre um padrão visual elementar em coordenadas cartesianas e outro em coordenadas polares é a definição matemática do estímulo. Isto é, em um sistema de coordenadas cartesianas, a modulação de luminância do estímulo (grade senoidal) é definida em relação aos eixos x e $y$, enquanto que em um sistema de coordenadas polares, a modulação da luminância é definida em termos de $\theta$ e $r$, onde $\theta$ representa a variável angular e $r$ a variável radial. Outros detalhes sobre estímulos elementares em coordenadas cartesianas e polares podem ser encontrados em Santos e Simas (Santos, 1999; Santos \& Simas, 2001a; Simas \& Santos, 2002a, 2002b).

Atualmente existem evidências, baseadas em estudos psicofísicos e neurofisiológicos, de que freqüências espaciais em coordenadas cartesianas são processadas nos estágios iniciais da visão, isto é, em neurônios do córtex visual primário, V1 (R. L. De Valois \& K. K. De Valois, 1988; Manahilov \& Simpson, 2001; Wilkinson et al., 2000; Wilson \& Wilkinson, 1998; Wilson, Wilkinson \& Asaad, 1997). Por outro lado, resultados psicofísicos em humanos (Wilkinson, Wilson \& Habak, 1998; Wilson \& Wilkinson, 1997, 1998; Wilson et al., 1997), neurofisiológicos em primatas (Bruce, Desimone \& Gross, 1981; Desimone, 1991; Desimone \& Schein, 1987, Gallant et al., 1996) e imagem de ressonância magnética funcional em humanos (Wilkinson et al., 2000) destacam a importância das vias intermediárias (p.ex., área visual V4) e áreas mais avançadas (p.ex., córtex visual ínfero-temporal, IT) do sistema visual no processamento de estímulos espaciais em coordenadas polares. Alguns desses trabalhos destacam que a área V4 pode formar o principal estágio intermediário da visão da forma de V1 para IT (Heywood, Gadotti \& Cowey, 1992; Merigan, 1996; van Essen, Anderson \& Felleman, 1992; Wilkinson et al., 2000; Young, 1992). Assim, é possível que a filtragem e integração de padrões radiais e angulares em coordenadas polares ocorram em áreas visuais extra-estriatais ( $p$. ex., V4 e IT). Inclusive, o estudo de Wilkinson et al. (2000) com a técnica de imagem de ressonância magnética funcional em humanos reforçou os estudos psicofísicos com humanos e neurofisiológicos com primatas que demonstraram inicialmente que estímulos em coordenadas polares são processados em áreas visuais extra-estriatais (p. ex., V4 e IT). O termo processamento da informação visual pode ser entendido como a descrição de um processo desencadeado pelo SVH ao detectar um estímulo levando em consideração os aspectos sensoriais e perceptivos.

Recentemente, Santos e Simas (2002b) mensuraram pela primeira vez as curvas de resposta ao contraste para filtros de freqüências radiais de 1 e 4 cpg com o método psicofísico de somação de supralimiares aliado ao método da escolha forçada. Os resultados demonstraram somações máximas de limiar de contraste na freqüência de teste dos filtros de 1 e $4 \mathrm{cpg}$ circundadas por inibições nas freqüências vizinhas às freqüências radiais de teste de cada filtro. Estes resultados foram favoráveis à existência de canais de freqüências radiais de banda-estreita no SVH através do processo de somação ou inibição na faixa de freqüências radiais estudada.

Por sua vez, Simas e colaboradores realizam uma série de estudos visando caracterizar a resposta do $\mathrm{SVH}$ para canais ou filtros de freqüências angulares de banda-estreita (Simas \& Dodwell, 1990; Simas et al. 1992; Simas \& Santos, 2002b). No estudo mais recente, Simas e Santos estimaram com o método de somação de supralimiares a curva de resposta ao contraste para os filtros de freqüências angulares de $1,2,3,4,8,16$ e 24 ciclos $/ 360^{\circ}$. Os resultados 
demonstraram efeitos de somação absoluta e relativa nas freqüências angulares de teste dos filtros circundadas por inibições. Os autores defenderam a existência de canais ou filtros seletivos para faixas específicas de freqüências angulares.

A largura de banda de passagem de um filtro se refere à função de resposta para as freqüências espaciais desse filtro. Dentro desse contexto, a curva de resposta para um filtro transmite informações (a) sobre a banda (ou bandas) de passagem do filtro, (b) sobre as frequiências em que o filtro responde menos ou não responde (deixa passar alguma coisa ou não deixa passar nada), e (c) sobre aquelas freqüências que estão fora da faixa de resposta do filtro. É importante destacar que, quanto mais estreita a largura da banda, mais alta sua seletividade à freqüência espacial e vice-versa.

O presente estudo deu continuidade aos experimentos que investigam o processamento visual humano de padrões de freqüências angulares em coordenadas polares. Neste sentido, procuramos investigar mecanismos ou canais putativos sintonizados para freqüências angulares definidas em coordenadas polares com bandas de passagem estreitas centradas em 32, 48, 64 e 96 ciclos $/ 360^{\circ}$. O objetivo principal foi caracterizar com o método psicofísico de somação de supralimiares a existência de canais de bandaestreita para filtros de freqüências angulares na alta freqüência. Os estudos de Simas e colaboradores investigaram mecanismos putativos centrados em freqüências angulares baixas e médias (Simas \& Dodwell, 1990; Simas et al., 1992; Simas \& Santos, 2002b).

O método psicofísico de somação de supralimiares é uma adaptação do método psicofísico de somação de sublimiares de Kulikowski e King Smith (1973). No estudo original de Kulikowski e King Smith, eles empregaram 1/2 do contraste necessário para alcançar o limiar de contraste dos respectivos estímulos de teste e de fundo (ruído). A finalidade foi obter, eventualmente, a somação do contraste da freqüência de teste com o contraste da freqüência de fundo e alcançar, desse modo, o limiar de detecção. Nos experimentos com o método de somação de supralimiares, ao invés de se utilizar somação de resposta com níveis de contraste sublimiar para mensurar o máximo da função, utiliza-se somação de resposta de supralimiar de contraste, onde a freqüência de teste é somada pixel a pixel às freqüências de fundo que são apresentadas a alto contraste. Nesse, enquanto o contraste da freqüência de teste é variado de acordo com a sensibilidade do voluntário, o contraste da freqüência de fundo é fixado acima do limiar (isto é, supralimiar). Desta forma, se um estímulo contendo apenas uma das freqüências de fundo é comparada a um estímulo em que a mesma freqüência de fundo é somada a freqüência de teste, a única forma de diferenciar entre os dois estímulos é detectar a presença da freqüência de teste em um destes. Informações mais detalhadas sobre este paradigma podem ser encontradas em Santos e Simas (Santos, 1999; Santos \& Simas, 2002a, 2002b; Simas \& Dodwell, 1990; Simas \& Santos, 2002a, 2002b).
Durante o processo de filtragem, três tipos de respostas podem ocorrer: (a) aquelas em que determinadas freqüências de fundo podem se somar à freqüência de teste do filtro facilitando a sua detecção (efeito de somação); (b) aquelas em que as freqüências de fundo e de teste podem não se somar, não facilitando nem dificultando sua detecção (efeito independente); ou, (c) aquelas em que as freqüências de fundo podem inibir a percepção da freqüência de teste do filtro, dificultando a sua detecção (efeito inibitório). A sensibilidade ao contraste da freqüência de teste em função das freqüências de fundo, usando como referência o limiar absoluto de detecção da freqüência de teste, indicará a independência dos mecanismos de deteç̧ão ou os efeitos de somação ou inibição.

A interpretação dada ao paradigma de somação de supralimiar é: (a) se a superposição da freqüência de teste do filtro à frequeiência de fundo facilita ou diminui o valor de contraste absoluto necessário à detecção, isto pode ser porque a freqüência de teste e a freqüência de fundo estão sendo processadas pelo mesmo mecanismo ou canal; (b) se a superposição da freqüência de teste à freqüência de fundo dificulta ou aumenta o valor de contraste absoluto, isto podem ser porque elas são processadas por canais distintos que interagem entre si possivelmente de forma antagônica; (c) se a superposição da freqüência de teste à freqüência de fundo não facilita e nem dificulta o valor de contraste absoluto (efeito independente), a freqüência de teste e a freqüência de fundo possivelmente estão sendo processadas por canais distintos que não interagem entre si.

Participaram do experimento seis adultos, um homem (DHE) e cinco mulheres (DKO, MSM, MM, KMC, RMT), com acuidade visual normal ou corrigida na faixa etária de 20-25 anos. A participação foi voluntária mediante assinatura do termo de consentimento livre e esclarecido.

\section{Equipamentos e Estímulos}

Foi utilizado o sistema desenvolvido no próprio laboratório, que compreende um monitor de vídeo SONY-BVM1910, com entrada "RGBsync" entrelaçada e interfaciado a um microcomputador 486 de $30 \mathrm{MHz}$ através de uma placa de aquisição Data-Translation DT-2853. Um programa escrito em linguagem $\mathrm{C}$ foi desenvolvido no próprio laboratório para executar os experimentos. Uma cadeira giratória foi fixada a $150 \mathrm{~cm}$ da tela do monitor de vídeo. Uma tábua cinza foi situada acima do monitor de vídeo para onde o voluntário, entre uma apresentação e outra, deveria fixar o olhar com o objetivo de diminuir os efeitos de pós-imagem. A luminância média foi de $6,9 \mathrm{~cd} / \mathrm{m}^{2}$ ajustada por um fotômetro ASAHI PENTAX SPOTMETER, com precisão de um grau. A luminância mínima foi de $6,2 \mathrm{~cd} / \mathrm{m}^{2} \mathrm{e}$ a máxima de $7,5 \mathrm{~cd} / \mathrm{m}^{2}$. O ambiente do laboratório era cinza para melhor controle da luminância.

Os estímulos de freqüências angulares foram gerados em tons de cinza e apresentados em tempo real no monitor. 
Todos os estímulos também eram circulares com um diâmetro de 7,25 graus de ângulo visual a $150 \mathrm{~cm}$ de distância da tela (Figura 1).

As estimativas foram feitas utilizando pares de estímulos, estímulo de teste e estímulo de fundo. O estímulo de teste era composto pela freqüência de teste do filtro angular $\left(32,48,64\right.$ ou 96 ciclos $\left./ 360^{\circ}\right)$ somada a uma das freqüências angulares de fundo de $1,2,3,4,5,6,7,8,9,10,12,16,24,32,48,64$ ou 96 ciclos $/ 360^{\circ}$. O estímulo de fundo consistia na própria freqüência de fundo isolada. A Figura 1 ilustra exemplos de pares de estímulos de freqüências angulares utilizados, por exemplo, para mensurar o filtro angular de $32 \operatorname{ciclos} / 360^{\circ}$.

\section{Procedimento}

O procedimento para medir cada filtro angular utilizando o método de somação de supralimiares aliado ao método da escolha forçada consistiu na apresentação sucessiva simples do par de estímulos: um contendo a freqüência

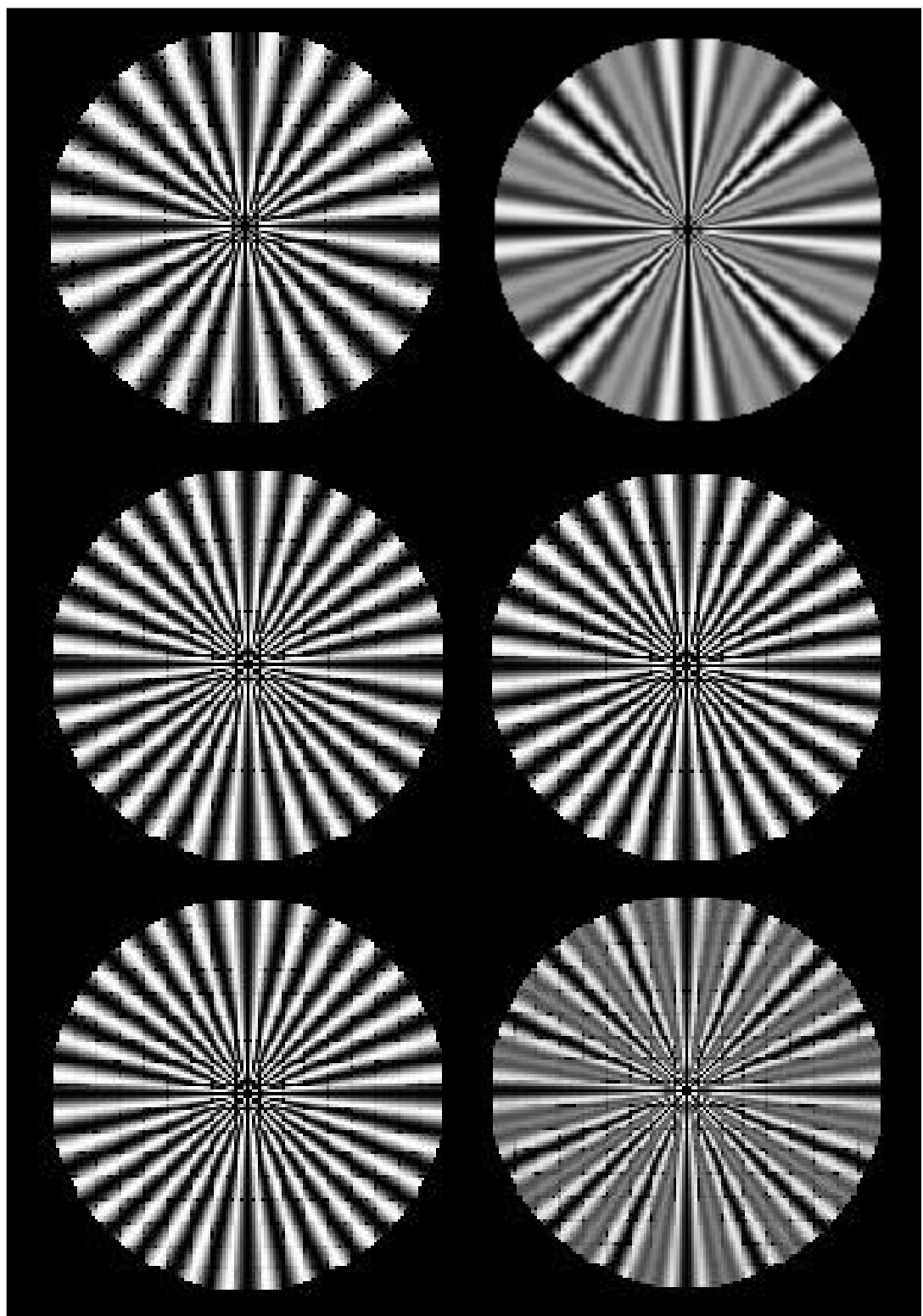

Figura 1. Exemplos de pares de estímulos de freqüências angulares utilizados para estimar o filtro angular de 32 ciclos. Acima à esquerda a freqüência de 24 ciclos e à direita 24 ciclos somada à 32 ciclos, ao centro à esquerda 32 ciclos e à direita 32 ciclos somada à 32 ciclos e embaixo à esquerda 48 ciclos e à direita 48 ciclos somada à freqüência de 32 ciclos. angular de teste do filtro somada à freqüência angular de fundo (estímulo de teste) e o outro contendo uma das 17 freqüências angulares isolada (estímulo de fundo). $O$ contraste da freqüência de fundo foi mantido constante e correspondia a cinco vezes o limiar absoluto de detecção da freqüência de teste do filtro angular (F32, F48, F64 e F96), tanto no estímulo de teste como no estímulo de fundo. Somente o contraste da freqüência de teste era variado, diminuindo ou aumentando de acordo com o método da escolha forçada (Santos \& Simas, 2002b; Santos et al., 2003, 2004; Wetherill \& Levitt, 1965). A tarefa do participante foi escolher dentre os dois estímulos qual deles continha a freqüência angular de teste do filtro somada à frequiência angular de fundo. $\mathrm{O}$ critério adotado para variar o contraste da freqüência de teste era o de três acertos consecutivos para decrescer uma unidade e um erro para acrescer da mesma unidade $(0,08 \%)$. Este procedimento experimental se baseia no cálculo da probabilidade de acertos consecutivos por parte do voluntário, ou seja, em cerca de 100150 apresentações de escolhas entre os dois estímulos, o estímulo de teste é percebido $79 \%$ das vezes pelo voluntário.

A seqüência temporal foi iniciada com um sinal sonoro seguido imediatamente pela apresentação do primeiro estímulo por $2 \mathrm{~s}$, seguido de um intervalo entre estímulos de $1 \mathrm{~s}$, seguido pela apresentação do segundo estímulo por $2 \mathrm{~s}$ e da resposta do participante. A ordem de apresentação dos estímulos era aleatória. Se a resposta do participante fosse correta, ela era seguida por outro sinal sonoro e um intervalo de $3 \mathrm{~s}$ para a seqüência temporal se repetir. Em outras palavras, $3 \mathrm{~s}$ era o intervalo entre tentativas, independente da resposta (ou escolha) ser correta ou não. Os sinais sonoros que indicavam o início da apresentação do par de estímulos e a escolho correta eram diferentes. A sessão experimental variava em duração dependendo dos erros e acertos do participante até proporcionarem um total de dez máximos e dez mínimos conforme requerido para o final automático da mesma. Sua duração era da ordem de 10-20 min.

Os participantes foram orientados antes da sessão a pressionar a barra de espaço quando julgassem que o estímulo de teste tivesse sido apresentado primeiro e 
qualquer tecla acima da barra de espaço quando julgasse que o mesmo tivesse sido apresentado em segundo lugar, isto é, após o estímulo de fundo.

A ordem de mensuramento das freqüências angulares para cada curva foi aleatorizada por sorteio pelo voluntário antes de mensurar cada uma das três curvas para cada um dos quatros filtros. Todas as estimativas foram feitas à distância de $150 \mathrm{~cm}$, com visão binocular.

Cada uma das 17 sessões experimentais requeridas para medir cada um dos quatros filtros (F32, F48, F64 e F96) foi realizada pelo menos três vezes, em dias diferentes, por pelo menos três participantes. Portanto, um total de nove curvas foi coletado para cada filtro gerando uma amostra de 180 valores de máximos e mínimos para cada um dos 17 pontos estimados (cada freqüência de fundo correspondeu a um ponto e a uma sessão experimental). A distribuição dos seis participantes entre os filtros mensurados foi a seguinte: F32 (DHE, DKO, MSM), F48 (DHE, DKO, MM), F64 (DHE, MM, RMT), F96 (DHE, KMC, RMT).
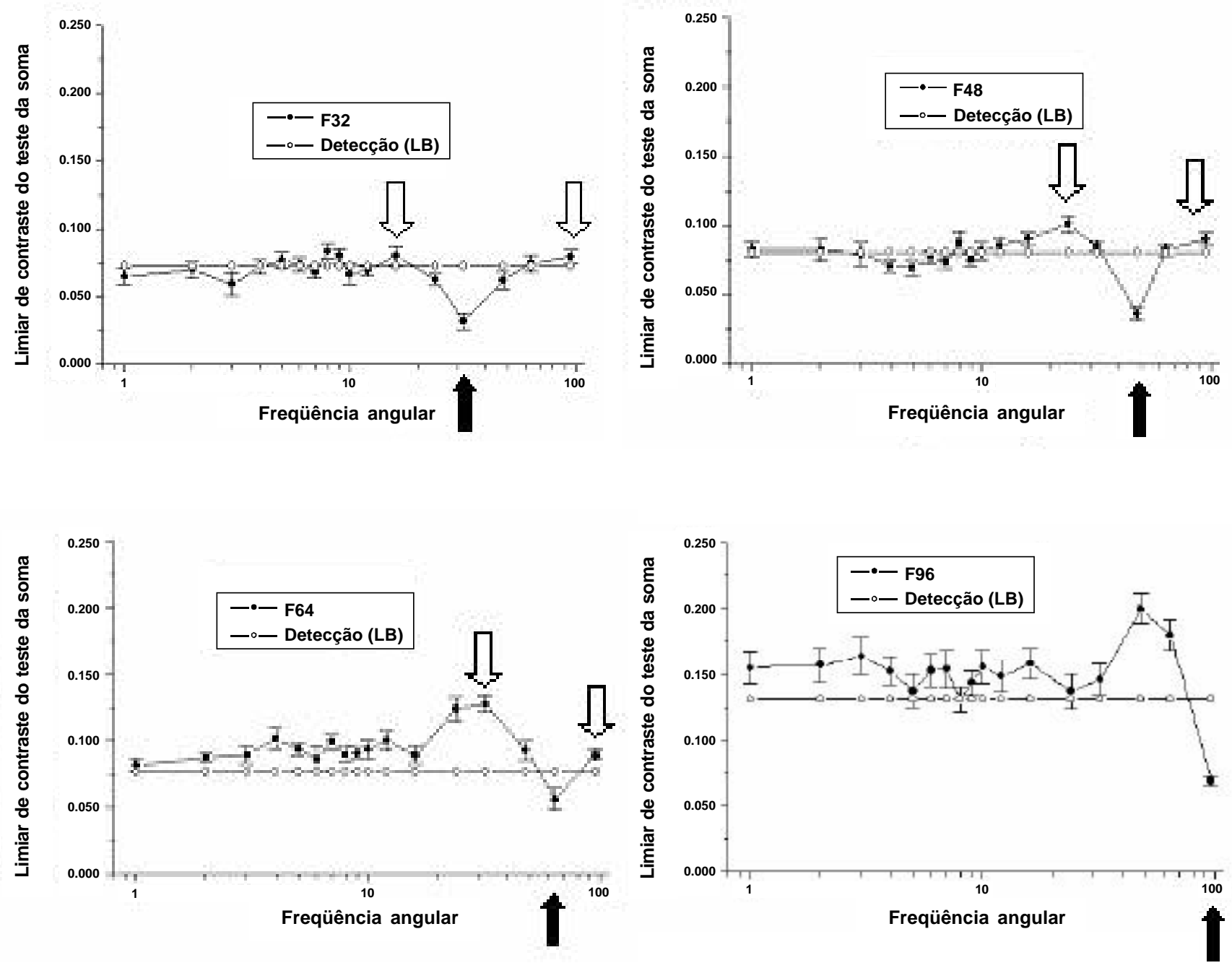

Figura 2. Curvas de resposta ao contraste da freqüência angular de teste de 32 (acima à esquerda), de 48 (acima à direita), de 64 (abaixo à esquerda) e de 96 ciclos $/ 360^{\circ}$ (abaixo à direita) somada às respectivas freqüências angulares de fundo representadas na abscissa. As setas pretas indicam os limiares de somação e as brancas os de inibição. 
confiança dos erros padrões da média assim corrigidos se sobrepõem até mesmo pela metade, ainda assim um teste $t$ Student para amostras correlacionadas pode revelar diferenças entre as médias que podem ser significantes ao nível de $p<0,05$. Nos casos em que os erros padrões da média não se sobrepõem, as diferenças das médias podem ser significantes até ao nível de $p<0,001$.

Os resultados, portanto, mostram que os filtros de freqüências angulares F32, F48, F64 e F96 apresentam efeito de somação máxima em suas respectivas freqüências angulares de teste, i.e. $32,48,64$ e 96 ciclos $/ 360^{\circ}$ (vide Figura 2, efeito indicado por setas pretas), circundado de inibição nas freqüências angulares vizinhas (vide Figura 2 , inibição indicada por setas brancas). Um teste $t$-Student para amostras correlacionadas nos pontos de somação máxima comparados às respectivas linhas de base (i.e, limiares de detecção dos estímulos testes isolados) mostrou significância de $p<0,001$ para todos os casos em que as freqüências angulares de teste e de fundo eram iguais. Para F32, a média foi igual a 0,0318 vs $0,0743, t=18.75, d f=179$; para $\mathrm{F} 48$, igual a 0,0365 vs $0,0814, t=18.40, d f=179$; para F64, igual a 0,0563 vs $0,0767, t=9.77, d f=179$ e para F96, igual a 0,0693 vs $0,1308, t=19.84, d f=179$.

As razões entre somação máxima e linhas de base foram da ordem de 2,3; 2,2; 1,4 e 1,9 para os filtros de freqüências angulares F32, F48, F64 e F96, respectivamente. Por outro lado, a inibição máxima foi geralmente observada nos harmônicos das freqüências angulares de teste: por exemplo, 16 e 64-96 ciclos no caso do filtro de freqüência angular F 32; 24 e 96 ciclos no caso do filtro de freqüência angular F48; 32 e 96 ciclos no caso do filtro de freqüência angular F64 e 48 ciclos no caso de freqüência angular F96. Isto indica claramente os efeitos de filtragem. Em outras palavras, a banda de passagem de cada filtro angular apresentou alta seletividade, o que implicou em somação máxima na freqüência de teste de cada filtro, circundada em ambos os lados por efeito de inibição nos harmônicos e circunvizinhanças (vide setas brancas e pretas na Figura 2).

\section{Discussão}

Os resultados com filtros de freqüência angular F $32, \mathrm{~F} 48$, F64 e F96 apresentam somação máxima na freqüência de teste de cada filtro angular mensurado. Observa-se ainda uma banda-estreita de passagem ou filtragem bem delimitada para todos filtros investigados, o que de certa forma mostra uma alta seletividade do SVH para freqüências angulares.

Estes resultados são consistentes com os dados de Simas e colaboradores (Simas \& Dodwell, 1990; Simas et al., 1992; Simas \& Santos, 2002b), que apresentaram somações máximas de limiares de contraste nas freqüências angulares de teste de todos os filtros angulares circundadas por inibições nas freqüências angulares vizinhas às freqüências angulares de teste. Esse efeito foi verificado para todos os filtros estudados.

Um outro aspecto importante é que os nossos resultados com filtros de frequiências angulares F32, F48, F64 eF96 também demonstram efeitos de somação secundários para freqüências angulares baixas, quando a freqüência de teste do filtro é somada a freqüências angulares de fundo muito mais baixas. Por exemplo, o filtro angular F32 apresentou efeitos de somação relativos em freqüências de fundo de $3 \mathrm{e}$ 10 ciclos $/ 360^{\circ}$; por sua vez, o filtro angular $\mathrm{F} 48$ apresentou efeitos de somação relativos nas freqüências de fundo de 4,5 e 7 ciclos $/ 360^{\circ}$; enquanto, o filtro angular F64 apresentou somação relativa na freqüência de fundo de 6 e 16 ciclos $/ 360^{\circ}$; e por último, o filtro angular F96 apresentou efeito de somação relativa em frequiência de fundo de $5,8 \mathrm{e}$ 24 ciclos $/ 360^{\circ}$. Estes resultados demonstram evidências para interações entre canais de freqüências espaciais em coordenadas polares. Resultados nesta direção já foram descritos na literatura para grades senoidais em coordenadas cartesianas (K. De Valois, 1977; Tolhurst, 1972; Tolhurst \& Barfield, 1978) e também para estímulos de freqüências radiais em coordenadas polares (Santos \& Simas, 2002b). Por exemplo, alguns trabalhos com grades senoidais encontraram casos de sensibilidade aumentada para freqüências espaciais à cerca de uma oitava da freqüência da grade de teste (K. De Valois, 1977; Tolhurst, 1972; Tolhurst \& Barfield, 1978). Esses autores discutiram que isso constitui evidência para interações entre canais de freqüências espaciais. Alguns resultados também mostram interações entre o fundamental e o terceiro harmônico (Pollen \& Ronner, 1982). Apesar destes resultados serem com padrões cartesianos ao invés de polares, são interessantes na medida em que os nossos resultados para filtros angulares em coordenadas polares também apresentam interação entre canais de freqüência angular de banda estreita. Estes resultados também demonstram que os canais para freqüências angulares não são totalmente independentes. Em outras palavras, um modelo de canais múltiplos não implica necessariamente que em qualquer condição de estimulação e teste o sistema visual se comporte como completamente composto por canais de freqüências espaciais fortemente independentes (Sekuler, 1974).

É importante, ainda, destacar que embora os resultados apresentados aqui e os encontrados na literatura (Simas \& Dodwell, 1990; Simas et al., 1992; Simas \& Santos, 2002b) demonstrem a existência de filtros de freqüências angulares em todo o espectro estudado (1-96 ciclos $\left./ 360^{\circ}\right)$, ainda é muito cedo para definir o número de canais putativos existentes para frequiências angulares no SVH. Os estudos psicofísicos demonstram a presença de filtros de freqüências angulares, entretanto a definição da largura da banda de passagem depende da realização de estudos eletrofisiológicos. Por exemplo, os estudos psicofísicos e eletrofisiológicos com grades senoidais defendam a idéia geral de que a freqüência espacial é processada por canais independentes e seletivos para aproximadamente uma oitava de freqüência espacial (Blakemore \& Campbell, 1969a, 1969b; R. L. De Valois \& K. K. De Valois, 1988; Sekuler, 1974). Isto implica que as características dos canais seletivos a grades senoidais na visão central humana cobrem uma faixa de 3-4 oitavas (Blakemore \& Campbell, 1969b; 
R. L. De Valois \& K. K. De Valois, 1988; Sekuler, 1974). Uma oitava é uma alteração na freqüência por um fator de dois, isto é, oitavas de freqüência espacial são incrementos iguais em uma escala logarítima.

Estudos com estímulos definidos em coordenadas cartesianas e em coordenadas polares envolvem conceitos distintos, como por exemplo: (a) grade senoidal é um estímulo dimensional (i.e., a freqüência varia em função da distância do observador), enquanto que a freqüência angular é adimensional (i.e., a freqüência não varia em função da distância do observador); (b) grades senoidais e freqüências angulares são processadas por áreas visuais distintas; (c) a luminância da grade senoidal é definida em função de $x$, enquanto que a luminância da freqüência angular é definida em função do ângulo. Então, a freqüência angular é discreta e sempre dada por número inteiro de ciclos por $360^{\circ}$. Neste contexto, acreditamos que filtragem e detecção de estímulos em coordenadas polares (p.ex., freqüência angular) pode coexistir com a perspectiva tradicional de filtragem de estímulos em coordenadas cartesianas (p.ex., grade senoidal). Em outras palavras, dependendo da configuração matemática e física do estímulo (p.ex., uma face ou um padrão circularmente simétrico) é muito mais adequado e econômico para o sistema visual fazer uma decomposição ou o processamento da informação espacial em termos que poderiam ser melhor descritos em um sistema de coordenadas polares (p.ex., freqüência angular ou radial) do que em um sistema de coordenadas cartesianas (grade senoidal). Estes aspectos e a alta sensibilidade do SVH para freqüências angulares quando comparada a grades senoidais (Simas et al., 1992) contribuem para um modelo em coordenadas polares.

A noção de canal ou filtro supõe que um estímulo apresentado no campo visual não estimula todos os neurônios corticais que recebem informações daquela área da retina, mas apenas um grupo de neurônios seletivos para uma dimensão do estímulo, por exemplo, freqüência espacial. Assim, se um segundo estímulo que difere do primeiro ao longo da faixa de freqüência (ou uma outra dimensão qualquer) é apresentado na mesma área, ele ativará um grupo de neurônios diferente do primeiro. Em outras palavras, estímulos diferentes são processados por grupos ou subunidades de neurônios diferentes, os quais formam a base dos canais ou filtros.

Talvez o pressuposto básico aqui seja a idéia de que, dependendo do filtro espacial (grade senoidal, radial ou angular), mecanismos diferentes interdependentes e intradependentes sejam ativados (efeito de inibição, somação e independência dos canais para freqüências espaciais radiais). Os filtros investigados aqui podem não ser como um filtro ideal (p.ex., um filtro óptico ou um circuito elétrico sintonizado que deixa a quantidade desejada inalterada e reduz todos os outros valores a zero), porém fica a idéia que o sistema de filtragem executado pelo SVH é muito robusto, o que nos leva a crer que a circuitaria visual dispõe de mecanismo para se ajustar a qualquer tipo de padrão de freqüências espaciais em coordenadas polares ou cartesianas. Desta forma, o sistema visual extrai as características necessárias para reconhecer os detalhes que diferenciam um padrão de outro de acordo com as "suas necessidades".

A filtragem espacial realizada pelo SVH parece ter uma função crucial, pois permite ao sistema lidar seletivamente com uma diversidade muito grande de características simples e complexas de objetos em estágios anteriores que possivelmente são integradas em estágios posteriores, permitindo a reconstrução e interpretação do objeto ou da cena visual, que possivelmente resulta naquilo que denominamos de percepção visual da forma. A grande maioria de nossas idéias sobre o mundo é baseada na visão.

O objetivo principal do sistema visual talvez seja representar o ambiente visual da maneira mais concreta para a realidade na qual o sistema foi adaptado. Há quem acredite, que o sistema visual exista para derivar da imagem a informação que nós precisamos e não simplesmente para recriar a imagem projetada na retina (Braddick, Campbell \& Atkinson, 1978).

Em síntese, estes resultados fornecem evidências psicofísicas para a existência de filtros distintos sintonizados para percepção de padrões de freqüências angulares em coordenadas polares. Entretanto, por outro lado, estes resultados sobre filtragem de freqüências angulares não podem provar por si só que o sistema visual utiliza este tipo de filtro para processar e reconhecer objetos ou uma cena visual. Portanto, o fato de o SVH ser sensível a estímulos desta natureza, por si só é um indício forte da importância destes estímulos no processamento visual de forma.

A idéia geral baseada nos nossos dados e nos dados encontrados na literatura, é que se não existem mecanismos distintos seletivos a freqüências angulares, radiais, radiais/ angulares acopladas e grade senoidal, pelo menos devem existir mecanismos distintos ou áreas distintas para estímulos em coordenadas polares e para estímulos em coordenadas cartesianas.

Todos os resultados apresentados e discutidos neste trabalho são consistentes com a possibilidade de que estímulos em coordenadas polares são importantes para caracterizar a resposta do $\mathrm{SVH}$.

\section{Referências}

Amidor, I. (1997). Fourier spectrum of radially periodic images. Journal of the Optical Society of America A, 14, 816-826.

Blakemore, C., \& Campbell, F. C. (1969a). Adaptation to spatial stimuli. Journal of Physiology, 200, 11-12p.

Blakemore, C., \& Campbell, F. C. (1969b). On the existence of neurons in the human visual system selectively sensitive to the orientation and size of retinal images. Journal of Physiology, 203, 237-260.

Braddick, O., Campbell, F. W., \& Atkinson, J. (1978). Channels in vision: Basic aspects. In R. Held, H. W. Leibowitz \& H. L. Teuber (Eds.), Handboob of Sensory Physiology: Vol. 5. Perception (pp. 3-38). New York: Springer-Verlag. 
Bruce, C. J., Desimone, R., \& Gross, C. G. (1981). Visual properties of neurons in a polysensory area in superior temporal sulcus of the macaque. Journal of Neurophysiology, 46, 369-384.

Campbell, F. W., \& Robson, F. G. (1968). Application of the Fourier analysis to the visibility of gratings. Journal of Physiology, 197, 551-566.

De Valois, K. (1977). Spatial frequency adaptation can enhance contrast sensitivity. Vision Research, 17, 1057-1065.

De Valois, R. L., \& De Valois, K. K. (1988). Spatial Vision. New York: Oxford University Press.

Desimone, R. (1991). Face-selective cells in the temporal cortex of monkeys. Journal of Cognitive Neuroscience, 3, 1-8.

Desimone, R., \& Schein, S. J. (1987). Visual properties of neurons in area V4 of macaque: Sensitivity to stimulus form. Journal of Neurophysiology, 57, 835-867.

Gallant, J. L., Brau, J., \& van Essen, D. C. (1993). Selectivity for polar hyperbolic, and cartesian gratings in macaque visual cortex. Science, 259, 100-103.

Gallant, J. L., Connor, C. E., Rakshit, S., Lewis, J. W., \& van Essen, D. C. (1996). Neural responses to polar, hyperbolic, and cartesian gratings in area $\mathrm{V} 4$ of the macaque monkey. Journal of Neurophysiology, 76, 2718-2739.

Gilinski, A. S. (1968). Orientation-specific effects of patterns of adapting light on visual acuity. Journal of the Optocal Society of America, 58,13-18.

Heywood, C. A., Gadotti, A., \& Cowey, A. (1992). Cortical area $\mathrm{V} 4$ and its role in the perception of color. Journal of Neuroscience, 12, 4056-4065.

Kulikowski, J. J., \& King-Smith, P. E. (1973). Spatial arrangement of line, edge and grating detectors relealed by sub-threshold summation. Vision Research, 13, 1455-1478.

Maffei, L., \& Fiorentini, A. (1973). The visual cortex as a spatial frequency analyzer. Vision Research, 13, 1255-1267.

Manahilov, V., \& Simpson, W. A. (2001). Energy model for contrast detection: Spatial-frequency and orientation selectivity in grating summation. Vision Research, 41, 1547-1560.

Menees, S. M. (1998). The effect of spatial frequency adaptation on the latency of spatial contrast detection. Vision Research, 38, 3933-3942.

Merigan, W. H. (1996). Basic visual capabilities and shape discrimination after lesions of extrastriate area $\mathrm{V} 4$ in macaques. Visual Neuroscience, 13, 51-60.

O'Donnell, B. F., Potts, G. F., Nestor, O. G., Stylianopoulos, K. C., Shenton, M. E., \& McCarley, R. W. (2002). Spatial frequency discrimination in schizophrenia. Journal of Abnormal Psychology, 111 (4), 620-625.

Pollen, D. A., \& Ronner, S. F. (1982). Spatial computation performed by simple and complex cells in the visual cortex of the cat. Vision Research, 22, 101-118.

Sachs, M. B., Nachmias, J., \& Robson, J. G. (1971). Spatial frequency channels in human vision. Journal of the Optical Society of America, 61, 1176-1186.

Santos, N. A. (1999). Sistema visual humano: Curvas de sensibilidade e filtragem de freqüências angulares, radiais e radial/angulares acopladas. Tese de Doutorado não-publicada, Curso de PósGraduação em Neurociências e Comportamento, Instituto de Psicologia, Universidade de São Paulo, SP.

Santos, N. A., \& Simas, M. L. B. (2001a). Função de sensibilidade ao contraste: Indicador da percepção visual da forma e da resolução espacial. Psicologia: Reflexão E̊ Crítica, $14(3)$, 589-597.
Santos, N. A., \& Simas, M. L. B. (2001b). Percepção e processamento visual da forma: Discutindo modelos teóricos atuais. Psicologia: Reflexão E゚ Crítica, 14(1), 151-160.

Santos, N. A., \& Simas, M. L. B. (2002a). Função de sensibilidade ao contraste: Indicador da percepção visual da forma e da resolução espacial. Psicologia: Reflexão e Crítica, 14(3), 589-597.

Santos, N. A., \& Simas, M. L. B. (2002b). Percepção e processamento visual da forma em humanos: Filtros de freqüências radiais de 1 e 4 cpg. Psicologia: Reflexão e Crítica, 15(2), 383-391.

Santos, N. A., Simas, M. L. B., \& Nogueira, R. M. T. B. L. (2003). Processamento visual da forma em idosos: Curvas de limiar de contraste para freqüências angulares e senoidais. Psicologia: Reflexão e Crítica, 16(2), 271-277.

Santos, N. A., Simas, M. L. B., \& Nogueira, R. M. T. B. L. (2004). Comparison of angular frequency contrast sensitivity in young and older adults. Brazilian Journal of Medical and Biological Research, 37, 375-378.

Sekuler, R. (1974). Spatial vision. Annual Review of Psychology, 25, 195-232.

Simas, M. L. B., \& Dodwell, P. C. (1990). Angular frequency filtering: A basis for pattern decomposition. Spatial Vision, 5, 59-74.

Simas, M. L. B., Frutuoso, J. T., \& Vieira, F. M. (1992). Inhibitory sidebands in multiple angular filters in the human visual system. Brazilian Journal of Medical and Biological Research, 25, 919-923.

Simas, M. L. B., \& Santos, N. A. (2002a). Contrast sensitivity to radial frequencies modulated by $\mathrm{Jn}$ and jn Bessel profiles. Brazilian Journal of Medical and Biological Research, 35, 13571366.

Simas, M. L. B., \& Santos, N. A. (2002b). Narrow-band 1, 2, 3, 4, 8, 16 and 24 cycles $/ 360^{\circ}$ angular frequency filters. Brazilian Journal of Medical and Biological Research, 35, 243-253.

Tolhurst, D. J. (1972). Adaptation to square-wave gratings: Inhibition between spatial frequency channels in the human visual system. Journal of Physiology, 226, 231-248.

Tolhurst, D. J., \& Barfield, L. P. (1978). Interactions between spatial frequency channels. Vision Research, 18, 851-858.

van Essen, D. C., Anderson, C. H., \& Felleman, D. J. (1992). Information processing in the primate visual system: An integrated systems perspective. Science, 255, 419-423.

Verrall, S. C., \& Kakarala, R. (1998). Disk-harmonic coefficients for invariant pattern recognition. Journal of the Optical Society of America A, 15, 389-401.

Wetherill, G. B., \& Levitt, H. (1965). Sequential estimation of points on a psychometric function. The British Journal of Mathematical and Statistical Psychology, 48, 1-10.

Wilkinson, F., Wilson, H. R., \& Habak, C. (1998). Detection and recognition of radial frequency patterns. Vision Research, 38 , 3555-3568.

Wilkinson, F., James, T. W., Wilson, H. R., Gati, J. S., Menon, E. S., \& Goodale, M. A. (2000). An fMRI study of the selective activation of human extrastriate form vision areas by radial and concentric gratings. Current Biology, 10, 1455-1458.

Wilson, H. R., Levi, D., Maffei, L., Rovamo, J., \& De Valois, R. (1990). The perception of form: Retina to striate cortex. In S. W. Spillmann \& J. S. Werner (Eds.), Visual perception: The Neurophysiological Foundation (pp. 231-271). New York: Academic Press. 
Wilson, H. R., \& Wilkinson, F. (1997). Evolving concepts of spatial channels in vision: From independence to nonlinear interactions. Perception, 26, 939-960.

Wilson, H. R., \& Wilkinson, F. (1998). Detection of global structure in glass patterns: Implications for form vision. Vision Research, 38, 2933-2947.

Wilson, R. W., Wilkinson, F., \& Asaad, W. (1997). Concentric orientation summation in human form vision. Vision Research, 37, 2325-2330.
Young, M. P. (1992). Objective analysis of topological organization of the primate cortical visual system. Nature, 358 , 152-155.
Recebido: 15/06/2004

$1^{a}$ revisão: $08 / 07 / 2005$

$2^{a}$ revisão: $12 / 09 / 2005$

Aceite final: 09/06/2006 